\title{
EDITORIAL
}

\section{Standing up to antimicrobial resistance}

\author{
Recent outbreaks involving multidrug-resistant bacteria have prompted governments \\ to alter the regulations governing antibiotic sales. By combining the new regulations \\ with calls for new drug development and dispensing regimes, can antibiotic resistance \\ be managed?
}

The WHO has declared antibiotic resistance to be one of the three greatest threats to human health. Two examples show that the numbers involved are staggering: methicillin-resistant Staphylococcus aureus (MRSA) is responsible for 100,000 infections in the United States annually, leading to nearly 20,000 deaths, and multidrugresistant Mycobacterium tuberculosis affects over 500,000 individuals worldwide.

Indeed, widespread antibiotic use provides a potent selective force for antibiotic-resistant bacteria and is therefore often blamed for the rise of resistance. The medical community has long been aware that stemming the rise of resistant bacteria requires prudent use of antibiotics, and during the past year several governments have also shown an increased awareness by implementing, or planning to implement, regulations that limit access to antibiotics. The spread of the New Delhi metallo- $\beta$-lactamaseresistance gene in Enterobacteriaceae in India and its rapid dissemination to the United Kingdom led the Drug Controller General of India (DCGI) to introduce new rules aimed at stopping the sale of antibiotics without a prescription. In Brazil, new regulations from the National Health Alert Agency (or Anvisa) that similarly require a prescription for the sale of antibiotics were prompted by an outbreak of drugresistant Klebsiella pneumoniae that has killed more than 40 people this year.

Furthermore, the US Food and Drug Administration is readying guidelines to restrict the use of antibiotics in livestock to the treatment of infections. In the United States, antibiotics are given to livestock even in the absence of infection, as prophylaxis and to promote growth, and $70 \%$ of the antibiotics used annually are consumed by farm animals. The new guidelines, which are due to be released early next year, are opposed by the food industry, which claims that there is no evidence to link the use of antibiotics in farm animals with antibiotic resistance in human pathogens. However, those who oppose the use of antibiotics in farm animals feel that the guidelines, which will be voluntary, do not go far enough. Many US hospitals will not wait for the guidelines; by the summer of 2010, 300 hospitals had switched to serving meat from antibiotic-free animals to prevent the potential transfer of antibiotic-resistance genes to patients. The use of antibiotics in farm animals is an issue that could be resolved by the Atlantic Task Force on Antimicrobial Resistance, an organization founded in 2009 that aims to bring together the relevant authorities and promote understanding of antimicrobial-resistance programmes. It is due to report in the spring of 2011, with the hope that a closer working relationship can be forged between the United States and Europe to reduce the spread of resistance.

To target organisms that have already acquired resistance, new drugs need to be developed. This year has seen two important calls for increased antimicrobial drug discovery. In the United Kingdom, the British Society for Antimicrobial Chemotherapy announced its 'Urgent Need' initiative to regenerate antibacterial drug discovery, and in the United States the Infectious Disease Society of America has released its ' 10 by 2020' programme, which calls for the development of ten new antibiotics by 2020 . The market for antimicrobials is expected to be US $\$ 40.3$ billion in 2015 , providing an incentive to continue the development of new drugs. When new drugs are launched, strict dispensing guidelines must be implemented to decrease the appearance of resistance. One example is the antimalarial drug artemisinin. In 2006, the makers of artemisinin agreed to phase out artemisinin monotherapy, following the recommendation of the WHO. Artemisinin is now sold only as part of a combination therapy, greatly decreasing the odds that artemisinin resistance will arise.

Antibiotic resistance will always be a problem because bacteria continuously find ways to avoid the effects of antimicrobial drugs. Although the current situation is far from good, international recognition of the problem and the political will to address the free dispensing of antibiotics, combined with improved prescription strategies, will hopefully slow the spread of resistance. Increased interest in the development of new antimicrobials could lead to new drugs that would reach the market before the end of the decade. So, the problem will not go away, but could we see a time when we can at least manage antimicrobial resistance? 